\title{
Intubation unter ungünstigen Rahmenbedingungen Methoden zur Überprüfung der korrekten Tubuslage in der Notfallmedizin
}

\author{
M. Sroka, I. Hornke, Institut für Anästhesiologie und operative Intensivmedizin, Klinikum Stadt Hanau, Hanau \\ (Direktor: Prof. Dr. med. Peter M. Osswald)
}

NOTFALLMEDIZIN 2003; 29: 260-262

\begin{abstract}
„Die endotracheale Intubation ist der Goldstandard zur Sicherung der Atemwege“ (1). Dies gilt umso mehr für Notfallpatienten. Leider müssen jedoch gerade unter Notfallbedingungen häufig Intubationen unter widrigen äußeren Umständen durchgeführt werden, so zum Beispiel bei einem noch eingeklemmten Unfallopfer. Diese schlechten äußeren Rahmenbedingungen tragen dazu bei, dass die endotracheale Intubation nicht immer, beziehungsweise nicht auf Anhieb gelingt. Daher sind Vorkehrungen zum Management dieser Situationen zu treffen und zum Beispiel alternative Materialien des erweiterten AirwayManagements vorzuhalten. Nach erfolgter endotrachealer Intubation stell sich die Frage: Liegt der Tubus wirklich in der Trachea? Ösophageale Tubusfehllagen sind die häufigsten Komplikationen der notfallmäßigen Intubation. Bleiben sie unerkannt und unkorrigiert, so hat dies deletäre Folgen für den Patienten. Welche Methoden stehen uns unter klinischen Bedingungen sowie in der Notfallversorgung zur Verfügung, die korrekte Tubuslage zu überprüfen? Dieser Artikel will eine aktuelle Übersicht liefern und die besondere Rolle der $\mathrm{CO}_{2}$-Detektion darstellen.
\end{abstract}

( rundsätzlich müssen wir zwischen apparativen und nicht apparativen Methoden zur Kontrolle der Tubuslage unterscheiden. Historisch bedingt spielen gerade in der Notfallmedizin die nicht apparativen Methoden eine herausragende Rolle.

\section{Nicht apparative Methoden Auskultation}

„Die Auskultation der Lungen alleine ist ungeeignet zur Unterscheidung zwischen trachealer und ösophagealer Intubation“(2). In der anästhesiologischen und notfallmedizinischen Literatur herrscht Uneinigkeit bezüglich der Reihenfolge der Auskultation. Einige Autoren empfehlen mit der Auskultation des Epigastriums zu beginnen und dann bei fehlendem Anhalt für eine Fehllage die Auskultation der Lungen anzuschließen (3). Dieses Vorgehen deckt sich mit den aktuellen Empfehlungen zur Reanimation (4). Die Auskultation der Lungen liefert wei- tere wertvolle Informationen, mit ihr kann eine einseitige, das heißt meist zu tiefe Intubation ebenso wie ein Pneumothorax erkannt werden. Ihre Sensitivität zum Ausschluss einer Fehlintubation darf jedoch nicht überbewertet werden. Da die Auskultation nach erfolgter Intubation zum Standard gehört, kann ein Verzicht darauf unter Umständen als Verletzung der Sorgfaltspflicht bewertet werden. Trotzdem sind unter Bedingungen des Rettungsdienstes viele Störfaktoren der Auskultation denkbar, die die Sensitivität der Methode weiter reduzieren.

\section{Wasserdampfkondensation}

Bei trachealer Tubuslage lässt sich regelmäßig eine atemsynchrone Wasserdampfkondensation an der Tubusinnenwand beobachten. Diese kann jedoch auch bei ösophagealer Tubuslage auftreten. Daher ist die Wasserdampfkondensation kein Beweis für die korrekte Tubuslage. Ihr Fehlen sollte jedoch als Hinweis für eine eventuelle Fehllage gewertet werden und eine entsprechende Überprüfung nach sich ziehen.

\section{Thoraxwandbewegung}

Die Kontrolle der Thoraxwandbewegung unter Beatmung wird ebenfalls als Methode zur Kontrolle der Tubuslage empfohlen (5). Eine klinische Inspektion ist immer erforderlich, zumal sich dadurch zum Beispiel Thoraxwandverletzungen erkennen lassen. Sich bei der Frage der korrekten Tubuslage jedoch alleine auf diese Beobachtung zu verlassen, erscheint mehr als fragwürdig.

\section{Oesophageal Detection Device (ODD)}

Hierbei handelt es sich um einen Ballon (z.B. Ambu, TubeChek) der in entleertem Zustand auf den Tubuskonnektor des Endotrachealtubus aufgesteckt wird. Bei trachealer Lage des Tubus ist die Luftaspiration problemlos möglich. Bei ösophagealer Lage kommt es zu einer Verlegung des Tubus durch die Ösophagusschleimhaut. Neben diesem Ballonsystem existiert auch noch ein Spritzenmodell. Das Wirkprinzip ist das gleiche. Anatomische Korrelate sind die von Knorpelspangen offen gehaltene Trachea und der Bindegewebs-/ Muskelschlauch der Speiseröhre, der unter Sog kollabiert (6). Die ODD haben wegen ihrer einfachen Anwendung bei hoher Sensitivität Einzug in die internationalen Empfehlungen zur Reanimation gehalten (4).

Neben diesen klinischen Methoden werden noch das Gefühl für die Compliance der Lunge über den Beatmungsbeutel das erforderliche 
Volumen zum Blocken des Cuffs sowie der Luftausstrom aus dem Tubus bei Thoraxkompression als Möglichkeiten der Lagekontrolle erwähnt. Diesen Methoden ist eine geringe Sensitivität und Spezifität gemeinsam, sie sind daher als alleinige Bestätigung der korrrekten Tubuslage ungeeignet $(7,8)$.

\section{Apparative Methoden zur Kontrolle der Tubuslage Pulsoxymetrie}

Die Pulsoxymetrie gehört inzwischen nahezu flächendeckend zur Ausstattung des Rettungs- und Notarztdienstes. Einzelne Autoren bewerten die Pulsoxymetrie extrem hoch. „Die Pulsoxymetrie ist zur Erkennung von Narkosezwischenfällen nützlicher als die Kapnometrie“ (2). Zur Erkennung einer Tubusfehllage ist die Pulsoxymetrie jedoch den Methoden der $\mathrm{CO}_{2}$-Detektion unterlegen. Jeder klinisch Tätige kennt den späten Sättigungsabfall bei einem gut präoxygenierten Patienten. Außerdem kann ein Abfall der Sättigung nach Narkoseeinleitung zahlreiche Ursachen haben. Die Pulsoxymetrie ist zwingend bei der Einleitung einer Narkose, insbesondere unter präklinischen Bedingungen, zu fordern. Ihre Aussagekraft bezüglich einer eventuellen Tubusfehllage ist jedoch nicht ausreichend beziehungsweise $\mathrm{zu}$ spät. Sie zeigt erst die sekundären Folgen der Tubusfehllage, nämlich die Hypoxämie.

\section{$\mathrm{CO}_{2}$-Indikatorsysteme,}

\section{z.B. EasyCap,}

Es existieren verschiedene einfache qualitative Systeme zum Nachweis von Kohlendioxid in der Ausatemluft. Diese Systeme gleichen einem Beatmungsfilter und werden in den Ausatemstrom eingeschliffen. Durch einen Farbumschlag wird $\mathrm{CO}_{2}$ qualitativ angezeigt. Trotzdem konnten sich diese kolorimetrischen Systeme in verschiedenen Untersuchungen bezüglich Sensitivität und Spezifität nicht durchsetzen (1).

\section{Apparative Methoden der $\mathrm{CO}_{2}$-Detektion}

Kapnometrie und Kapnografie sind bereits seit Jahren klinisch etabliert und haben ihre Sensitivität bezüglich der Erkennung von Tubusfehllagen bewiesen. Nicht ohne Grund schreiben die anästhesiologischen Fachgesellschaften und Berufsverbände diese zwingend am Narkosearbeitsplatz vor (9). Auch die Neufassung der DIN 75079 (10) sieht für das Notarzteinsatzfahrzeug ein Kapnometer verpflichtend als Ausstattungsgegenstand vor. Eine aktuelle Untersuchung (11) zeigt jedoch für Baden-Württemberg, dass nur rund ein Drittel aller arztbesetzten Rettungsmittel Equipement zur $\mathrm{CO}_{2}$-Detektion mitführt. Beide apparativen Methoden der $\mathrm{CO}_{2}$-Detektion ermöglichen auch unter ungünstigen äußeren Umständen eine sichere Aussage über die Lage des Endotrachealtubus. Als beweisend für die korrekte intratracheale Tubuslage gilt der (möglichst kontinuierliche, beziehungsweise für mindestens sechs Atemzüge) Nachweis von Kohlendioxid. Bei einer Fehlintubation wird kein Kohlendioxid nachweisbar sein. Selbst bei Patienten, die große Mengen an kohlensäurehaltigen Getränken zu sich genommen haben, bleibt die positive $\mathrm{CO}_{2}$-Antwort auf wenige Atemzüge/Beatmungen beschränkt. Daher sollte auch eine einmalig positive und im Verlauf sinkende $\mathrm{CO}_{2}$ Fraktion die besondere Aufmerksamkeit des Notarztes und des Rettungsdienstpersonals auf sich ziehen.

Bei der Kapnometrie wird der exspiratorische Kohlendioxidanteil gemessen und numerisch angezeigt. Die Kapnografie liefert zusätzlich Informationen über den Verlauf der $\mathrm{CO}_{2}$-Abatmung, der graphisch dargestellt wird. Physikalisch beruhen diese Messungen auf dem Prinzip der Infrarotspektroskopie. Dieses Messverfahren ist in zwei unterschiedlichen Gerätetypen realisiert worden. Hier sind Haupt- und $\mathrm{Ne}$ benstromverfahren zu unterscheiden. Beim Hauptstromverfahren befindet sich der Messfühler zwischen Tubus und Beatmungsschlauch. Die Messung erfolgt also direkt im Ausatemstrom des Patienten. Beim Nebenstromverfahren wird kontinuierlich eine kleine Gasfraktion patientennah abgesaugt und in einer patientenfernen Messkammer analysiert. Beide Verfahren haben spezielle Vor- und Nachteile, die der anästhesiologischen Fachliteratur zu entnehmen sind.

\section{Rolle der $\mathrm{CO}_{2}$-Detektion \\ für den Rettungsdienst}

Haupteinsatzgebiet der $\mathrm{CO}_{2}$-Detektion, sei es mittels Kapnometrie oder Kapnografie im Rettungsdienst ist die Kontrolle der Tubuslage. Die Rahmenbedingungen des Rettungsdienstes, wie Lärm oder Vibrationen, erfordern (wie auch in der Klinik) zusätzliche Hilfsmittel zur Verifizierung der korrekten Tubuslage. Die Kontrolle allein mit dem Stethoskop, oder anderen dargestellten Methoden, führt nur eingeschränkt und nicht sicher genug zum Ziel. Die auskultatorische Kontrolle des intubierten Patienten ist durch die Kapnometrie nicht ersetzbar und sollte wann immer möglich erfolgen, beziehungsweise schnellstmöglich nachgeholt werden. Die Anwendung des ODD sollte mehr Verbreitung finden.

Weitere Indikationen für die Kapnografie/-metrie im Rettungsdienst sind die Überwachung und Steuerung einer Beatmung. Hierbei ist die $\mathrm{CO}_{2}$-Detektion als ein „Backup-Alarm-System“ zu verstehen.

Häufige Komplikationen bei einer Beatmung - wie Abknickungen und Leckagen, Cuffhernien oder -defekte, eine unbeabsichtigte Extubation oder Diskonnektion - werden durch den fehlenden oder verminderten $\mathrm{CO}_{2}$-Rückstrom sofort erkannt. Auch technische Defekte oder der Ausfall des Beatmungsgerätes sind durch die Kapnometrie schnell und zuverlässig zu erfassen. Diese zweite unabhängige Überwachung führt zu einer erhöhten Sicherheit des beatmeten Patienten.

Der Stoffwechsel, die Zirkulation und die Ventilation beeinflussen die 
mit klinischen Parametern (zum Wohle des Patienten) interpretieren und in hilfreiche Handlungen umzusetzen wissen.

\begin{abstract}
Summary
"Endotracheal intubation is the gold standard for the securement of the airways" (1). This applies in particular to patients requiring emergency management. Unfortunately, emergency intubation frequently has to be done under less than optimal conditions, for example, in the case of an accident victim who is pinned down. Under such conditions, endotracheal intubation may not - or not immediately - be successful. This implies that appropriate measure should be taken to ensure that such a situation can be dealt with and, for example, that alternative materials for extended airway management are available. When endotracheal intubation has been successfully accom-
\end{abstract}

\section{Buch}

\section{Hilfestellung Umgang mit belastenden Ereignissen}

Mitchell JT, Everly GS. Streßbearbeitung nach belastenden Ereignissen. Edewecht: Verlagsgesellschaft Stumpf\&Kossendey, 1998

Igl und Müller-Lange kommt das Verdienst zu, das amerikanische Original übersetzt zu haben und in der 2. Auflage vorzulegen. Vor allem Einsatzkräfte (Rettungssanitäter, Polizei) sind gefährdet, nach einem Einsatz mit hoher psychischer Belastung eine posttraumatische Belastungsstörung zu entwickeln. Das vorliegende Buch - aus vielen Einzelberichten entstanden - ist als „Werkzeug“ gedacht, Hilfe zu leisten den Therapeuten, die einzeln oder in Gruppen posttraumatischen Stress professionell abbauen. Dabei gehen die Autoren systematisch vor, von der Erklärung der Grundbegriffe bis zur genauen Anwendung der erklärten Technik. Einzelne und unterschiedliche Behandlungsstrategien werden sehr ausführlich beschrieben bis hin zur Sitzordnung der Teilnehmer. Dabei wird auch auf besondere Bedingungen eingegangen. Manchmal ist die Sprache - wohl bedingt durch das amerikanische Original - zu blumig („Im folgenden soll nicht mit dem Finger auf Schuldige gezeigt werden“ S. 220), auch möchte man Inhalt und Text straffer, präziser, denn etwas zu häufige Wiederholungen erhöhen zwar den Lerneffekt, verhindern aber auch ein schnelles Lesen.

Dieses Buch wendet sich nicht nur an Ärzte und Psychologen, sondern eher noch an in die Behandlung einbezogenen Kollegen der Einsatzkräfte, im Buch als Peers bezeichnet. Ihnen wird die Lektüre eine wertvolle Hilfe sein.

Dr. med. Ulrich Rendenbach, Duderstadt plished, the question arises: is the tube actually in the trachea?. The most common complication of emergency intubation is the erroneous placement of the tube in the oesophagus. If this goes uncorrected, deleterious consequences for the patient ensue. What methods are available for checking the correct position of the tube under clinical and emergency conditions? The present article provides an up-to-date overview and describes the particular role of $\mathrm{CO}_{2}$ detection.

\section{Literatur}

1. Krier C, Georgi R. Airway-Management - Die Sicherung der Atemwege. Stuttgart/New York: Georg Thieme Verlag, 2001 2. Hartung HJ, Osswald PM, Petroianu G. Die Atemwege. Stuttgart: Wissenschaftliche Verlagsgesellschaft mbH, 2001

3. Anderson et. al. Assessing the position of the tracheal tube. Anaesthesia 1989; 44: 984-985

4. Guidelines 2000 for cardiopulmonary resuscitation and emergency cardiovascular care. An international consensus of science. Resucitation 2000; 46: 115-127

5. Schara. Rasche und sichere Überprüfung der Tubuslage. Anästh 1983; 32: 501

6. Pollard. A test to verity accurate placement of endotracheal tube, $7^{\text {th }}$ World Congress of Anaesthesia, Hamburg 1980

7. Caplan et al. Adverse respiratory events in anesthesia. Anesthesiology 1990; 72: 828-833

8. Hempelmann et. al. AINS, Band 3: Notfallmedizin. Stuttgart/New York: Georg Thieme Verlag, 1999

9. Ausstattung des anästhesiologischen Arbeitsplatzes - Fortschreibung der Richtlinien der Deutschen Gesellschaft für Anästhesiologie und Intensivmedizin und des Berufsverbandes Deutscher Anästhesisten. Anästhesiologie \& Intensivmedizin 1989; 30: 307-314

10. DIN Deutsches Institut für Normung e.V. DIN 75079.Berlin: Beuth Verlag, 08/2002

11. Genzwürker $\mathrm{H}$ et al. Erhebliche Unterschiede bei der Ausstattung von Notarzt besetzten Rettungsmitteln. Notfall- \& Rettungsmedizin 2002; 5 (6): 441-447

Weitere Literatur bei den Verfassern.

\section{Anschrift für die Verfasser}

Michael Sroka Klinikum Stadt Hanau

Institut für Anästhesiologie und operative Intensivmedizin

Leimenstr. 20

63450 Hanau

E-Mail: sroka@gmx.de 